\title{
El Escepticismo Radical de Pierre Gassendi Contra la Filosofía Natural de los Aristotélicos ${ }^{1}$
}

\author{
Leonel Toledo ${ }^{2}$ \\ Samuel Herrera ${ }^{3}$
}

\begin{abstract}
RESUMEN: Este artículo tiene como objetivo dar cuenta del escepticismo radical de Pierre Gassendi propuesto en el Exercitationes paradoxicae Adversus Aristoteleos (Disertaciones paradójicas contra los aristotélicos). Para dar cuenta de ello nuestro artículo se divide en tres momentos: Primero, damos cuenta de la estructura de la obra; segundo, se presentan los argumentos centrales propuestos por Gassendi para comprender su actitud crítica al escolasticismo. Y tercero, se muestra la crítica que realiza a la lógica aristotélica. La idea fundamental que sostenemos en este trabajo es que la actitud crítica y de libre pensador que caracterizó a Gassendi tuvo su origen directo en la actitud escéptica. Además, revisar su obra de juventud y su actitud filosófica nos permite comprender una parte importante del alcance del escepticismo en la reforma de la filosofía natural del siglo XVII.
\end{abstract}

PALABRAS CLAVES: Escepticismo. Filosofía natural. Reforma. Anti-aristotelismo. Antiescolasticismo. Siglo XVII.

\section{INTRODUCCIÓN}

Pierre Gassendi (1592-1655) concentra su escepticismo radical en las Exercitationes paradoxicae Adversus Aristoteleos (Disertaciones paradójicas contra los aristotélicos), con el afán de cuestionar la perspectiva escolástica de la filosofía natural. Se cuentan dos ediciones de la obra. La primera edición de las Disertaciones del año 1624, sólo contenía el libro primero, mientras la segunda edición fue extendida al libro segundo e incluida en en su Opera omnia de 1658.

\footnotetext{
${ }^{1}$ Artículo asociado al proyecto DI13-0060 de la Dirección de Investigación de la Universidad de La Frontera, Chile, titulado «La interpretación y recepción de la filosofía de Aristóteles en el Exercitationes paradoxicae contra Aristoteleos de Pierre Gassendi».

${ }^{2}$ Universidad Autónoma de Ciudad de México, México. E-mail: leontoledo@gmail.com

${ }^{3}$ Universidad de La Frontera, Temuco, Chile. E-mail: samuel.herrera@ufrontera.cl
} 
Las Disertaciones... es probablemente la obra menos estudiada de Gassendi. Generalmente se le considera un texto de importancia menor porque en él no encontramos los principales aportes de Gassendi al pensamiento moderno: En las Disertaciones...no aparece asumido su proyecto de atomismo enmarcado en un modelo de naturaleza teleomecanicista, o porque ellas son más bien un tratado eminentemente escéptico y, como tal, las estrategias discursivas son suficientemente conocidas como para merecer una atención más detallada.

En las páginas siguientes trataremos de dar cuenta del tipo de escepticismo que encontramos en dicha obra, y de ofrecer una interpretación sobre los elementos que componen las Disertaciones. Finalmente, haremos una breve evaluación del escrito gassendiano, señalando los alcances que consideramos más importantes.

\section{1.-ESQUEMA DE LA OBRA}

El plan inicial de la obra fue proyectado en siete libros. La publicación en 1624 del primer libro de las Disertaciones paradójicas contra los aristotélicos, y en parte fue motivada por un intento de formalizar las versiones libres y no autorizadas que circulaban entre sus estudiantes de la Universidad de Aix. El Segundo libro aparece mucho más tarde publicado en las obras completas de Gassendi, en 1658. De los restantes libros, la versión más correcta que tenemos hasta el día de hoy es que jamás llegaron a concretarse. (ROCHOT, 1944, p.19-21)

Específicamente, diversas consideraciones habrían retrasado al autor a publicar el Segundo libro. Por una parte, el contexto de censura en el cual se publicó el primer libro fue desmotivador para publicar el segundo. Recordemos que las normas de protección de todo aquello que estuviese fuera o en contra del escolasticismo fue una característica de aquellos días. Ya desde inicios del siglo XVII el parlamento antiguo de París erige a la doctrina aristotélica-escolástica como la única ortodoxa y reglamentaria. De hecho durante el mismo 1624 ocurre la querella contra Antoine de Villon, Jean Bitaud y Étienne de Clave quienes fueron acusados de antiaristotelismo (KHAN, 2002, p. 143-198). Y por otra, que a decir de algunos estudiosos de la obra gassendiana, hacia 1624, la mayor parte de las críticas que Gassendi podría hacer al aristotelismo de las escuelas ya formaba parte del contexto 
intelectual de la época: la insuficiencia de los términos y de las perspectivas escolásticas estaba, para esos ańos, claramente establecida.

Son ocho las disertaciones contenidas en el Primer libro, de las cuales podemos esquematizar las siguientes líneas críticas:

1) Disertación primera: La filosofía se ha vuelto una pura ciencia de las palabras, y nada tiene que ver con una investigación de la naturaleza a través de la experiencia (GASSENDI, 1959, p.44)

2) Disertación segunda: La filosofía escolástica, dedicada a desarrollar sus propios términos, se ha vuelto una especie de "prisión" intelectual. (GASSENDI, 1959, p.58-62)

3) Disertación tercera: No hay razones en la teología, en la filosofía natural, ni en la filosofía moral para preferir a la escolástica por sobre otras teorías. (GASSENDI, 1959, p.70-80)

4) Disertación cuarta: Siendo las mismas obras de Aristóteles sujetas a interpretación (algunas incluso se le adjudican falsamente), las verdaderas opiniones del Filósofo permanecen en la incertidumbre, esto acarrea numerosos conflictos al interior la misma secta aristotélica. (GASSENDI, 1959, p.94-112)

5) El corpus aristotélico muestra deficiencias (Disertación quinta), superfluidades (Disertación Sexta), errores, impiedades, falsedades (Disertación séptima) y contradicciones (Disertación Octava). (GASSENDI, 1959, p.114 y siguientes).

El Libro segundo trata de la dialéctica de los aristotélicos, contiene seis disertaciones cuyo contenido puede ser esquematizado del siguiente modo:

1) Disertación primera: La dialéctica artificial es innecesaria para el conocimiento de la naturaleza. (GASSENDI, 1959, p.234)

2) Observaciones críticas en torno a la doctrina de los universales, a las nociones de diferencia específica, género, especie, sustancia, accidentes, materia, forma (Disertación Segunda y Tercera.) y sobre las proposiciones (Disertación Cuarta). (GASSENDI, 1959, p.272 y siguientes)

3) Problemas en torno a la teoría de la demostración (Disertación quinta) (GASSENDI, 1959, 386 y siguientes) y a la ciencia en general (Disertación Sexta). (GASSENDI, 1959, p.434 y siguientes).

Tal y como Richard Popkin lo ha señalado, antes que una rigurosa exposición de las tesis aristotélicas en cuestión y su correspondiente crítica, 
las Disertaciones gassendianas son más bien un conjunto de reflexiones, donde los recursos más usados son, entre otros, los que se aproximan o tienen inspiración en el escepticismo de Sexto Empírico (POPKIN, 2003, p.120127), así como aquellas estrategias discursivas que fueron ampliamente esgrimidos por autores humanistas como Montaigne, y que encontramos también en el Quod nibil scitur de Francisco Sánchez (1581). Según Popkin (2003, p.55): "Montaigne extendió la crisis del conocimiento humanista: el tipo de duda engendrada por el redescubrimiento de una variedad de puntos de vista de pensadores de la antigüedad. A la luz de esa vasta diversidad de opiniones, ¿`ómo podemos decir cuál es la verdadera?”

En efecto, a lo largo de sus Disertaciones, Gassendi subraya la diversidad de opiniones y afirma que cada una de las disciplinas posee, en el mejor de los casos, su propia verdad, la que siempre queda reducida a hipótesis y a su propio método. Si esto es así, no podemos afirmar que haya una manera definitiva de conocer el mundo pues, “[...] la debilidad del espíritu humano es tal que no conoce las cosas en sí mismas, en su verdad: éste conforma, acerca de los temas, solamente conjeturas probables" (GASSENDI, 1959, p. 58).

\section{LAS CRÍTICAS GASSENDIANAS AL ESCOLASTICISMO}

Tanto en el Prefacio de las Disertaciones, como en la correspondencia de Gassendi, se encuentran ya los elementos principales que le guiarán en su parte "crítica" y, años después, en la dimensión constructiva de la filosofía natural: en este sentido será fundamental tanto la importancia de la libertad de pensamiento, así como su lectura de los escépticos Vives, Charron, del humanista Pico della Mirandola, entre otros. Así, el autor de las Exercitationes declara:

\footnotetext{
De todas las opiniones, ninguna me agradó tanto como la akatâlepsia sostenida por los Académicos y los pirrónicos. Después de haberme dado cuenta del abismo que existe entre el Genio de la Naturaleza y la mente humana, ¿qué otra cosa podría pensar, sino que las causas internas de los efectos naturales permanecen ocultas a la investigación? (GASSENDI, 1959, p.6).
}

Adoptando, además, el objetivo de la tranquilidad de ánimo que enseñaban los escépticos pirrónicos, Gassendi no deja de contrastarlo con la actitud de complacencia dogmática de las Escuelas y opina que ésta se ha vuelto perniciosa para la más elevada búsqueda de la sabiduría. Así, la primera 
queja contra los aristotélicos que nuestro autor enunciará en el Primer libro de sus Disertaciones, consiste en oponer la libertad de pensamiento a la "tiranía” y al "yugo" del escolasticismo:

La libertad del espíritu es algo más precioso que todo el oro del mundo y, bajo el impulso de la naturaleza, todo tiende a ser libre...entonces, nosotros los hombres, que buscamos la filosofía, ¿nos rebajaremos al punto de abrazar con tanto fervor y por voluntad propia tal servidumbre? En tanto que la filosofía promete una libertad por la que puede nacer la tranquilidad del alma y, con ella, la suprema felicidad, ¿qué locura furiosa nos conduce a practicar el culto de la filosofía bajo el yugo de tal servidumbre? (GASSENDI, 1959, p.58).

El tema de la servidumbre intelectual no es menor en el Primer libro de las Disertaciones y anuncia un tópico que, posteriormente, será fundamental durante la Ilustración. Gassendi insistirá en él a lo largo de la obra:

Sin embargo, algunos dicen: "¿no es una gran libertad la de poder pasar de la opinión de los nominalistas a la de los tomistas o los escotistas y adoptar la fórmula que más nos parezca verosímil?” Te pregunto, ¿qué libertad es esa? Es parecida a la de quienes, pudiendo correr como quieran dentro de una prisión, creen que son absolutamente libres. Pues no son sino los sectores de la prisión peripatética (GASSENDI, 1959, p.52).

Según Gassendi, los innumerables conceptos que se discuten en las artificiosas disputas escolásticas son el equivalente de estar bajo el yugo intelectual de una sola teoría, esto ha impedido la libertad necesaria para desarrollar perspectivas distintas en la filosofía natural. No sobra aquí recordar un célebre párrafo escrito más de cien años antes por Erasmo:

En estas refinadísimas sutilezas, entran en juego los diversos escolásticos. Te resultará más fácil salir del laberinto que del embrollo mental de realistas, nominalistas, tomistas, albertistas, escotistas. Y no he nombrado más que los principales. En todas ellas reina tal erudición y tal complejidad de dificultades que me imagino que los mismos apóstoles necesitarían otra vez del soplo del Espíritu Santo, si tuvieran que discutir hoy sobre estos temas con la nueva generación de teólogos. (ERASMO DE ROTTERDAM, 2003, p.110).

Notemos, a la vez, la diferencia entre los dos humanistas: mientras que Erasmo cuestionaba el escolasticismo a la luz de una especie de reforma 
religiosa, Gassendi, habiendo leído con cuidado a Montaigne, habría de buscar una reforma de la filosofía natural. Hacia 1624, entonces, era claro que la escolástica tampoco podía resolver satisfactoriamente las preguntas que se formulaban los pensadores de la modernidad temprana, tal y como lo veremos en el siguiente apartado.

Mientras tanto, es importante subrayar nuevamente el carácter escéptico de las Disertaciones: aunque sabemos que Gassendi adoptó, después, la hipótesis atomista (una suerte de epicureísmo), en 1624 el atomismo todavía no figura en su obra como la alternativa gassendiana a la escolástica. Encontramos, entonces, en las Disertaciones, a un pensador que desconfía profundamente de los alcances del conocimiento, de ahí también su advertencia acerca de adherirse a una sola doctrina; lo anterior lo acerca a los libertinos eruditos (BLOCH, 1971, p.288-292) y a la postura crítica de los siglos posteriores.

Tal vez en contra de la misma postura que después adoptará el pensador francés, los alcances (o si queremos, los aportes) más relevantes de las Disertaciones serán, precisamente, abrir el camino para la generación posterior de filósofos de la naturaleza, su ataque a un método científico común a todas las ramas del saber y la caracterización de la filosofía como una actividad libre, donde las asunciones están sujetas siempre a modificación; particularmente, debo recordar aquí el carácter aporético, no-sistemático de las Disertaciones: hacia 1624, Gassendi no se preocupaba, al parecer, por formular un dogma alternativo al de las escuelas, pues podía vivir bien con la indecisión, la equipolencia y la duda que enseñaban los escépticos.

\section{LAS DISERTACIONES SOBRE LA LÓGICA ARISTOTÉLICA}

La primera observación gassendiana en torno a la Lógica consiste en retomar la distinción entre la lógica natural y la artificial. La primera no será criticada, dado que es meramente entendida como el "sentido común"; dicha lógica "[...] no es otra cosa que la razón misma o una fuerza innata y un poder del entendimiento por el cual razonamos y discurrimos" (GASSENDI, 1959, p.234). El segundo tipo de lógica se refiere a la que es propiamente defendida en las escuelas.

Gassendi propone dos quejas principales en contra de la lógica de la escolástica y se pueden reformular de la siguiente manera: 
Primero, que la lógica artificial pretende ser una especie de metodología que dispone al entendimiento para lograr nuevos descubrimientos en la filosofía natural; de acuerdo al francés, esta asunción simplifica u olvida los procedimientos específicos de cada una de las ciencias en particular cuando se trata de encontrar nuevo conocimiento. Asimismo, en su segundo reclamo, nuestro autor afirma que conceptos como sustancia, accidente o las categorías no han sido extraídos de la experiencia y que, por tanto, no se refieren propiamente a los fenómenos del mundo. Ambas características tornan a la dialéctica aristotélica un conjunto de palabras, de nociones sin un auténtico valor epistémico:

¿Qué escalones producirá la lógica que me conducirán a un conocimiento completo de la naturaleza de una pulga, por no decir del sol? ¿Es cierto que está en la provincia de la lógica examinar, descubrir y, por tanto, explicar la naturaleza de las cosas, antes que la provincia de la física y de otras ciencias? (GASSENDI, 1959, p.238).

En efecto, para Gassendi, cada una de las ciencias tiene su propio método de descubrir y aumentar su propio conocimiento; tanto la regla o el criterio para sopesar lo verdadero y lo falso en cada una de ellas, varía según sean sus objeto de estudio en particular. Tal relativismo le conduce a negar la posibilidad de un método general o común para todas las disciplinas. Sin embargo, según Gassendi alguien puede plantear que la lógica artificial, aunque no aporta nuevo conocimiento de la naturaleza, sí nos provee de una descripción más clara y específica de las cosas sabidas. En tal caso:

Si alguien comete algún error sobre cierta materia, observarás que la causa no es su ignorancia de la lógica, sino que él no ha examinado la cuestión con suficiente cuidado; pero si él percibe el objeto claramente, apostaré mi vida a que él puede explicarlo claramente[...]consulta tantos artesanos como te plazca que no conozcan tu lógica o tu género o tu diferencia específica: ¡Dios inmortal!, ¡cuántas definiciones excelentes de todo tipo de cosas escucharás!. (GASSENDI, 1959, p.240).

La tesis que subyace a estas líneas es que la razón, la facultad cognitiva que por naturaleza poseemos los seres humanos, es suficiente para encontrar verdades y desarrollar conceptos rigurosos y claros. La lógica artificial de las escuelas, en tanto que impone sus propias categorías, resulta fútil, vana y perjudicial para el progreso de la filosofía natural. Más todavía, si aceptamos 
que una definición basada en materia, forma, sustancia y accidente es mejor formulada que una que no toma en cuenta tales nociones, el conocimiento de los fenómenos naturales quedará siempre aplazado y será suplantado (como lo es en la escolástica), por el arte de configurar definiciones tan sutiles como intrincadas:

Aun así dirás que, una vez que algún conocimiento de cierto tipo ha sido alcanzado, un lógico experto lo definirá mejor que alguien no educado (en la lógica). Pero entonces pregunto, ¿qué es lo que agrega al conocimiento de la cosa, una vez que éste ha sido logrado? La lógica debería ayudarnos con cosas que todavía no conocemos, pero cuando algo ha sido ya descubierto, ¿qué más puede agregar la lógica sino bagatelas? (GASSENDI, 1959, p.240-242).

Hemos mencionado líneas arriba la oposición relativista de Gassendi contra la doctrina de la dialéctica como método útil para todas las ciencias. Según nuestro autor, cada disciplina posee su método propio, sus medios particulares para distinguir tanto sus propios contenidos, como para determinar qué cosas cuentan como verdaderas en sus áreas específicas. Aunque podemos considerar que el francés es deudor de las advertencias baconianas sobre los obstáculos de las facultades cognoscitivas, debemos atender también la diferencia, puesto que no se trata aquí de encontrar un Novum organum, que corrija las insuficiencias del anterior. Para Gassendi, el conocimiento avanza como un conjunto de ciencias trabajando en su propio campo. Tal postura antecede la idea de crítica (ilustrada), en el sentido que pretende establecer los distintos alcances y límites de la razón, la cual debe actuar localmente:

Por ejemplo, deseo saber si es verdadero o falso que el acorde conocido como la octava suena del mismo modo si duplico el largo de una cuerda: ¿qué utilidad tiene para mí la lógica para determinar esto? Si quiero saber si es cierto o falso que una monarquía es preferible a otras formas de gobierno, ¿cómo esclarecerá esto la lógica? ¿No acudiríamos mejor al músico y al político o a otros artesanos, antes que al lógico para buscar la verdad en estos asuntos? (GASSENDI, 1959, p.240-242).

El resultado consistirá, entonces, en un esquema del conocimiento en el que las distintas regiones del saber operan bajo sus propias reglas. Si esto es así,

Cada ciencia tiene su propia verdad para ser conocida, cada una tiene sus propios parámetros para conocerla. La geometría tiene su cuadrante, 
la aritmética, sus cálculos; la física, los sentidos; la teología, su revelación; y otras ramas del conocimiento, tienen diferentes medios por los cuales lo verdadero debe ser distinguido de lo falso, distintas de las enseñanzas de la lógica artificial. (GASSENDI, 1959, p.248).

Si bien autores como Lutero, Erasmo o Montaigne habían criticado la pertinencia de la teología escolástica para las verdades de la fe, Gassendi, en sus Disertaciones, se propone dirigir sus ataques al aristotelismo, ahora en el ámbito de la lógica como método útil para la filosofía natural: su escepticismo le conduce a desconfiar de las pretensiones de universalidad de los enunciados de la ciencia, fundadas en la noción de diferencia, puesto que:

¿Cómo se llega al conocimiento de las diferencias? Obviamente, no pueden conocerlas salvo a través de los sentidos, los cuales pueden proveer una noticia de la diferencia común, a partir de la inducción basada en el cuadro de cada uno de los casos individuales. Sin embargo, junto con el hecho de que no se puede revisar cada caso en particular y, por tanto, no puede hacerse ningún enunciado universal absolutamente confiable; y que, además, todo conocimiento derivado de los sentidos es falible (y entonces, obviamente ningún conocimiento cierto de tal diferencia puede ser logrado a través de su operación); además de lo anterior, digo, ¿̇no es verdad (de acuerdo también con Aristóteles), que los sentidos están limitados solamente a la percepción de los accidentes?, ¿cómo lograrán pues llegar hasta la diferencia, que es parte de la esencia interior? (GASSENDI, 1959, p.400-402).

Es claro que los procesos cognoscitivos descritos por la tradición escolástica son rechazados: la posibilidad de aprender o concebir algo cierto y universal a partir de instancias particulares está ya fuera del alcance del intelecto, dado que las facultades de conocimiento avanzan (o se limitan) por la consideración de los casos particulares; además, considerando que el "material básico" que el intelecto posee para realizar sus operaciones son los datos sensoriales, ir más allá de las apariencias que éstos nos proveen nos conduce inevitablemente al terreno de la especulación sobre la naturaleza interior de las cosas y, en el mejor de los casos, al de la probabilidad. Gassendi entonces añade:

Tal vez dirás que las diferencias entre las cosas son conocidas por el entendimiento y no por los sentidos; pero, dado que, de acuerdo con el mismo Aristóteles nada hay en el intelecto que no haya estado primero en 
los sentidos, ¿cómo podrá el entendimiento concebir algo que no haya llegado a él a través de los sentidos? (GASSENDI, 1959, p.400-402).

El entendimiento, en todo caso, no podría aprender las diferencias y las esencias de los objetos mismos, puesto que éstas carecen de alguna fuente empírica. De ahí que, nuevamente, las nociones escolásticas estén reducidas a meras palabras y que deba buscarse el origen de tales ideas, no en el mundo externo, sino en la misma capacidad natural del intelecto de formularlas. ${ }^{4}$

Más todavía, continúa Gassendi, aun considerando que el intelecto pudiera abstraer, a partir de las cosas de la naturaleza, algo como sus diferencias y sus esencias, ¿cómo explicar, entonces, que tal capacidad funciona sólo en algunas ocasiones, y no en todas las instancias observables?

$\mathrm{Si}$, por el contrario, el entendimiento después de separar todos los fantasmas, es capaz de conocer las diferencias entre las cosas y, entonces, sus esencias, pregunto: ¿por qué las diferencias o las esencias de tales cosas como el imán, la anguila, y demás permanecen desconocidas? (GASSENDI, 1959, p.402).

En el párrafo anterior es clara la alusión gassendiana a las causas ocultas para ejemplificar los casos en los que el intelecto no parece aprender las esencias ni dar cuenta de los procesos naturales. Decir, por ejemplo, que los ejemplos anteriores "[...] se deben a cierta característica de la materia y de la forma que no es evidente", es no explicar nada, exponiendo lo oscuro por lo más oscuro. Gassendi insistirá en que, tal incapacidad de la teoría peripatética parece presentarse más bien en la mayoría de las cosas que pretendemos conocer: no tenemos una descripción adecuada y satisfactoria del modo en que la forma se une con la materia, cuál es su origen y cómo opera, cómo se dispone la materia en los casos específicos y cuáles son las reglas que dicha unión sigue al conformar los objetos. Así, fenómenos como el magnetismo y la bioluminiscencia son más que simples mirabilia: constituyen una razón más para establecer la insuficiencia de la ciencia escolástica y promover nuevas vías de explicación en la filosofía natural.

Pero, insiste Gassendi, algunos creen que podemos conocer al menos una diferencia en el caso del hombre que es racional. Suponiendo que este

\footnotetext{
${ }^{4}$ Esta tesis se desarrollará posteriormente en John Locke y, en general, las distintas versiones del empirismo moderno. (Cf. SPECHT, 1997, p. 238-239).
} 
principio racional o el origen de la racionalidad sea el alma, el filósofo francés se pregunta:

¿Conoces la naturaleza interna del alma racional? ¿Sabes cómo se origina?, ¿de qué sustancia está formada?, ¿qué tipo de existencia puede tener esta cosa desprovista de cuerpo?; siendo un espíritu, ¿cómo se une con el cuerpo?, ¿cómo origina las actividades corporales?, ¿cómo se disemina a través del cuerpo?, ¿cómo está presente en su totalidad en todo el cuerpo y también ella se encuentra entera en cualquier parte?, ¿̨cómo es indivisa, pero permanece intacta en el resto del cuerpo cuando un miembro de éste se elimina?[...]dado que es incorpórea, ¿cómo puede conocer las cosas corpóreas?, ¿cómo se forman las especies que se dice que le son transmitidas? (GASSENDI, 1959, p.404-406).

No dejemos de notar el cambio de estrategia en la crítica gassendiana: ahora se trata de reprochar la falta de explicación en torno a los procesos de comunicación entre el alma y el cuerpo; la crítica también prescribe, a su modo, la descripción de procesos operativos que la filosofía natural debe abordar. Tales preguntas, formuladas ya desde 1624, prefiguran, además, las críticas de Gassendi contra los postulados de la metafísica cartesiana.

Nuevamente, no será de mucha utilidad decir que el alma es la forma del cuerpo y que, de algún modo (que no conocemos) opera sobre el cuerpo. Finalmente, éste es el esquema que Gassendi antepone a las nociones fundamentales de la ciencia escolástica, mostrando que la mayoría de todas las cosas que se pretenden explicar quedan, en realidad, reducidas a debates sobre palabras. En el fondo, la naturaleza de las cosas permanece oculta.

Si la materia y la forma son inútiles para la filosofía natural, lo mismo sucede con la doctrina de los cuatro elementos:

¿Los cuatro elementos se encuentran en los hombres, en los peces, en las plantas, en los metales? ¿Pero en qué proporciones?, ¿qué compone (a los elementos)?, ¿en qué combinaciones? Para no extenderme sobre esto, si los cuatro elementos existen en una pulga, explícame, no de qué modo están allí, ni de qué manera están mezclados ni combinados, sino cuál es el método por el cual puedo conocer las cosas que quiero..(GASSENDI, 1959, p.490).

En suma: el método de la lógica escolástica queda reducido a controversias vacías sobre palabras y ha perdido, en todo caso, su "efectividad", al no establecer un programa de filosofía natural acorde a los parámetros que 
impone la nueva investigación sobre la función específica de las cosas del mundo, ni exponer claramente alguna descripción sobre sus componentes últimos.

Pero el escepticismo de Gassendi (1959, p.490) en 1624 es todavía más radical, pues, "[e]s claro que lo mismo puede decirse contra Demócrito, Platón y los demás. Por tanto, es patente que hasta aquí, no sabemos nada sobre las cosas naturales a través de los esfuerzos de toda la filosofía”.

\section{4.-NOTA FINAL}

Si bien es cierto que los ataques al escolasticismo datan de al menos un siglo antes que la redacción de las Exercitationes y, visto de manera superficial, el texto que hemos estudiado pertenece a esa bien establecida tradición escéptica sin más, creemos que Gassendi señala, junto con sus innumerables ataques al aristotelismo, distintas rutas de investigación que habrían de ser explorados por el mecanicismo del siglo XVII.

Hemos visto que, hacia 1624, Gassendi se propuso atacar los cimientos de la concepción de ciencia escolástica desde una postura escéptica, pero la discusión que desplegó en las Exercitationes contenía dificultades que, a nuestro juicio, definirían una nueva agenda para la filosofía natural. Esto se muestra especialmente porque al preguntar por los procesos específicos que tienen lugar en el intelecto o al reparar en el mecanismo que hace "funcionar" la estructura del mundo.

La desconfianza gassendiana por los sistemas de explicación válidos para todas las ciencias y su duda por los intentos de establecer certezas metafísicas, son temas presentes a lo largo de su obra: así lo muestra su posterior discusión con Descartes, por ejemplo, en torno al estatuto de las verdades matemáticas (OSLER,1997); Así como por el intento gassendiano de mantener toda teoría ontológica -incluso su propio atomismo- en el ámbito de las hipótesis, de lo opinable y probable, donde todo lo que se dice en torno a la naturaleza está sujeto a descripciones del mundo alternativas y contrarias. En palabras de Gassendi (1959, p.19), “[...] no hay proposición y opinión tan fuertemente establecida o tan atractiva, que su opuesta no se pueda mostrar igualmente probable o aun, en muchos casos, más probable".

¿Qué se ganó y qué se perdió con el escepticismo puesto en marcha contra la filosofía natural escolástica? Aquí depende de la valoración que 
estemos dispuestos a aceptar: por un lado, con la filosofía natural del s. XVII se "ganó" una descripción más simple, que permitió señalar y abordar numerosos efectos que habían sido atribuidos a las simpatías y antipatías, a los influjos estelares, a causas cuya explicación quedaba siempre aplazada. Por otro, se "perdió" una visión del mundo armonizada y coherente en casi todos sus niveles: desde el mundo sublunar hasta la divinidad misma. En este cambio de perspectiva ontológica y metodológica, es importante reconocer la labor del Gassendi más escéptico de las Exercitationes, para quien lo propio de la filosofía, es el ejercicio irrecusable de la crítica de las teorías desde las propias facultades naturales.

TOLEDO, Leonel; HERRERA, Samuel. The radical skepticism of Pierre Gassendi against the Aristotelian Natural Philosophy. Trans/Form/Ação, Marília, v. 37, n. 1, p. 187-200, Jan./ Abr., 2014

\begin{abstract}
The aim of this paper is to comprehend the radical skepticism of Pierre Gassendi in his book Exercitationes paradoxicae Adversus Aristoteleos(1624)(Paradoxical dissertations against the aristotelianism). To achieve this goal we explain his skepticism in three points: First, we show the book's structure; Second, we present the main arguments to understand his attitude against the scholasticism; and Third, we show his direct critic towards the Aristotelian logic. The fundamental idea that we hold in this article is that the skeptical attitude is the origin of critical aspects as free thinker, what distinguished all the gasssendi's philosophical work. Furthermore, to review his Exercitationes and the philosophical attitude allows us to appreciate an important part of the scope of skepticism in the reform of the seventeenth century natural philosophy
\end{abstract}

KEY WORDS: Skepticism. Natural philosophy. Reform. Anti-aristotelianism. Anti-scholasticism. Seventeenth century.

\title{
OBRAS CITADAS
}

BLOCH, Olivier René. La Philosophie de Gassendi. Nominalisme, Matérialisme et Métaphysique. La Haya: Martinus Nijhoff, 1971.

DE ROTTERDAM, Erasmo. Elogio de la locura. Madrid: Alianza, 2003.

GASSENDI, Pierre. Dissertations en forme de Paradoxes contre les Aristotléliciens/Exercitationes paradoxicae contra Aristoteleos. París: J. Vrin, 1959. 
KAHN, Didier. La condamnation des thèses d'Antoine de Villon et Etienne de Clave contre Aristote, Paracelse et les «cabalistes» (1624). Revue d'histoire des sciences. v. 55, n. 2. p. 143-198, 2002.

MONTAIGNE, Michel de. Apologie de Raimond Sebond. In: Essais II. París: Gallimard, 1962, p.138-351.

OSLER, Margaret. Volonté divine et vérité mathématique: le conflit entre Descartes et Gassendi sur le statut des vérités éternelles. In: MURR, Sylvia (Ed.). Gassendi et L'Europe. París: J. Vrin, 1997, p. 31-42.

POPKINS, Richard. The History of Scepticism from Savonarola to Bayle. Oxford: Oxford University Press, 2003.

SPECHT, Rainer. À propos des analogies entre les théories de la connaissance sensible chez Gassendi et Locke. In: MURR, Sylvia (Ed.). Gassendi et L'Europe. París: J. Vrin, 1997 p. $237-243$. 